\title{
FDG PET/CT in cardiac electronic devices infection: Now is the time to target guidelines implementation
}

\author{
François Rouzet, $M D, P h D,{ }^{\mathrm{a}, \mathrm{b}, \mathrm{c}}$ Fabien Hyafil, $\mathrm{MD}, \mathrm{PhD},{ }^{\mathrm{a}, \mathrm{b}, \mathrm{c}}$ and Dominique Le \\ Guludec, MD, PhD ${ }^{\mathrm{a}, \mathrm{b}, \mathrm{c}}$ \\ a Department of Nuclear Medicine, Bichat Hospital, Assistance Publique Hôpitaux de Paris \\ (APHP) and DHU FIRE, Paris, France \\ ${ }^{b}$ Paris Diderot University, Paris, France \\ ${ }^{c}$ INSERM U1 148, Paris, France
}

Received Feb 5, 2015; accepted Feb 5, 2015

doi:10.1007/s12350-015-0102-y

\section{See related article, pp. 787-798}

\section{CIEDS INFECTION: THE CLINICAL PROBLEM}

For the past 10 years, cardiovascular implantable electronic devices (CIEDs) have become a major tool in the therapeutic management of patients with cardiac diseases. The rise of cardiac resynchronization therapy associated or not with defibrillation as well as left ventricular assist devices had a considerable impact on the prognosis of patients with heart failure. ${ }^{1,2}$ Despite continuous improvement in implantation techniques, complications such as bleeding, pacing lead displacement, and infection occur at a steady or even increasing rate. Infection of CIEDs raises several specific issues. ${ }^{3}$ The first one is diagnostic: although patent local inflammatory changes of the generator pocket site make infection very likely, it is very difficult to distinguish between thrombus and vegetation when a mass adherent to a lead is detected by transesophageal echography (TEE). In addition, shadowing artifacts may alter the quality of images, and the absence of visualization of vegetation does not exclude lead infection. Overall, the definite diagnosis of infection requires positive microbiological cultures of samples taken within the pocket or

Reprint requests: Dominique Le Guludec, MD, PhD, Department of Nuclear Medicine, Bichat Hospital, Assistance Publique Hôpitaux de Paris (APHP) and DHU FIRE, 46 rue Henri Huchard, 75018

Paris, France; dominique.leguludec@bch.aphp.fr

J Nucl Cardiol 2015;22:800-3.

$1071-3581 / \$ 34.00$

Copyright (C) 2015 American Society of Nuclear Cardiology. from the hardware. A previous antibiotic treatment may cause false negative results. Otherwise, a follow-up long enough to rule out chronic infection is mandatory. Hence, the diagnosis of CIEDs infection may be very challenging, but any delay between the development of infection and hardware extraction is associated with a high risk of adverse events. Finally, when the infection of CIED seems likely, the removal of all hardware is mandatory, with all the consequences and decisions regarding the choice between percutaneous or surgical approach for leads extraction, monitoring of patients during the absence of CIED, and best timing for reimplantation of a new device.

\section{DIAGNOSTIC VALUE OF FDG PET/CT IN CIEDS INFECTION}

Since the application of FDG PET/CT in fever of unknown origin (FUO), a large body of literature has been provided supporting its use in cardiovascular infections, and more specifically in those related to CIEDs. Besides a few case reports and a pilot study, ${ }^{4}$ Ploux et al reported their experience in the clinically very relevant setting of FUO in patients with a pacemaker, but excluding those with unequivocal device infection. ${ }^{5}$ Although the sample size was limited (only 6 patients had definite CIED infection), they showed that the presence of FDG uptake along a pacing lead was specific of infection. Of note, in the control group consisting of 40 patients referred for oncologic indication, a positive signal was present on a lead in three of them $(7.5 \%)$.

A further validation of the diagnostic value of FDG PET/CT in larger population samples has been supplied by two additional studies. ${ }^{6,7}$ Sarrazin et al compared 
three groups of patients implanted with CIEDs: a group of patients suspected of infected device, a group of recently implanted device, and a third group of patients without infection. Non-attenuation-corrected images were used for final interpretation of infection and to determine the semi-quantitative ratio of maximum count rate of the device over mean count rate of lung parenchyma. The study evidenced the presence of mild post-operative residual inflammation up to 2 months after device implantation, whereas infected devices presented an uptake ratio significantly greater. Both the sensitivity and specificity were above $85 \%$ in a population at high prevalence of infection. Finally, those patients with a suspicion of infection but without FDG uptake presented a favorable outcome under antibiotic therapy, suggesting the absence of bacterial colonization of CIEDs. Noteworthy, no abnormal uptake was detected in the latter group of patients.

In a recent issue of the journal, Leccisotti et al investigated the impact of delayed ( 3 hours post-injection) acquisition versus conventional protocol. ${ }^{8}$ The difference in diagnostic performance between the two protocols was not significant on a patient-based analysis, although the sensitivity for the detection of lead infection was greater with delayed acquisitions. It should be noted, however, that the sensitivity in the conventional protocol was low.

In this issue of the journal, Tlili et $\mathrm{al}^{9}$ present the extension of the preliminary report previously published, ${ }^{5}$ by increasing the size of the study population (suspicion of CIEDs infection) as well as the control group (oncologic indication). The scans were performed under routine protocol, 1 hour after FDG injection and without specific diet designed to lower physiological cardiac uptake of the tracer. The retrospective analysis of FDG PET/CT was based mainly on a visual assessment of non-attenuation-corrected images in order to avoid correction artifact. The overall prevalence of infection was $45 \%$ (18/40) and most of infected devices were associated with patent signs (15/18) such as local inflammatory changes or vegetation attached to a pacing lead by TEE. The remaining study population corresponded to the indication of FUO in patients with CIED and negative or inconclusive TEE. In these conditions the diagnostic performance was in the range of previous studies, with a sensitivity of $83 \%$ and a specificity of $95 \%$. Although the additional value of a positive FDG $\mathrm{PET} / \mathrm{CT}$ in a patient with patent infection of the device is questionable, it is of major importance to identify correctly those with inconclusive clinical and echographic findings but in whom a conservative management would be harmful. On the other hand, the good sensitivity allows to consider that integration of 18FDG-PET results in the clinical decision making is likely to prevent the need for device removal in a substantial proportion of patients.

Taken together, these findings suggest that this imaging technique might find a role in the diagnostic work-up of patients suspected of CIED infection. However, some issues remain unresolved and require further investigation.

\section{FUTURE DIRECTIONS}

First of all, the optimal conditions of acquisition allowing to improve the image contrast and to better discriminate between positive and negative scans have to be determined. As suggested previously, ${ }^{8}$ delayed acquisitions may allow to decrease FDG activity surrounding the leads in their intravascular portion. It is also well acknowledged, thanks to the work performed in cardiac sarcoidosis ${ }^{10}$ and IE, ${ }^{11,12}$ that the physiological cardiac uptake of FDG alters the correct identification of an abnormal signal within the myocardium. It is therefore unfortunate that among all of the studies reported so far, only two ${ }^{6,8}$ included a highfat, low-carbohydrate diet in addition to the fasting period in the patients' preparation. Then, image analysis should be standardized regarding both the pattern and the quantification of the uptake.

Additionally, the impact of factors such as the presence of an antibiotic treatment and the infective agent needs to be evaluated more precisely. The initiation of antibiotic therapy and, if present, its duration prior to imaging is likely to alter the inflammatory response of the host and thus FDG uptake. Also, it is acknowledged that some bacteria strains may escape immune response either by producing a biofilm on prosthetic material, or by using an intracellular cycle, allowing them to be hardly detectable by immune cells. $^{13}$

An alternative approach of infection imaging relies on radiolabeled leukocytes (WBC) SPECT/CT. This technique demonstrated a good accuracy, in particular a high specificity, in prosthetic and native valve infective endocarditis. ${ }^{14,15}$ Quite recently, Erba et al reported the diagnostic value of WBC SPECT/CT in a series of 63 consecutive patients referred to a tertiary care center for suspicion of CIED infection. ${ }^{16}$ Their study substantiated the excellent specificity $(100 \%)$ as well as sensitivity (94\%) of the scan regarding both infection of the generator pocket or the leads. Our group recently compared the two approaches in the diagnosis of prosthetic valve endocarditis and we found that FDG PET/CT offered a higher sensitivity for the detection of infection than WBC SPECT/CT. ${ }^{17}$ A similar comparative study in the setting of CIED infection would help to define the role of each of these two imaging 
techniques in the overall diagnostic strategy. In particular, it is important to define the optimal patients' selection according to the probability of infection based on clinical evaluation and echocardiography results. It is clear that when the suspicion of infection is very low, the high sensitivity of PET/CT is a major strength to reliably rule out infection. Conversely, when the device removal would expose the patient to a life-threatening risk, the specificity of WBC is likely to prevail. This could be achieved by carrying out studies aimed at evaluating the real impact of imaging on patients' management. This is the only way to determine whether early diagnosis of infection or secondary septic location detection has an incremental value over management based on current recommendations.

Moreover, these studies should include the evaluation of the cost-effectiveness of FDG PET/CT. In a casecontrol study assessing the value of FDG PET/CT in patients with gram-positive bacteremia, Vos et al concluded that the incremental cost associated with PET was considered "acceptable" given the favorable prognostic impact. ${ }^{18}$ Furthermore, the major part of the additional cost was related to prolonged hospital stay in relation with treatment of metastatic infectious foci. Development of large international registries including patients with a suspicion of CIED infection would help to evaluate more precisely the use and impact of these new imaging techniques in this clinical situation.

Last update on the management of CIED infection released in 2010 by AHA does not even mention nuclear imaging techniques. ${ }^{13}$ Since then, the body of evidence supporting PET and SPECT in that domain grew up fast and they are now part of routine practice in many centers, so that it would be ethically questionable to assess their prognostic impact in the setting of a randomized trial. The level of evidence reached now prompted some experts to publish diagnostic flowcharts on CIEDs infection including nuclear imaging techniques. ${ }^{19}$ Efforts should now be directed toward the development of multicentre trials performed under standardized protocols regarding both acquisition and quantification of FDG uptake. This is the only way to achieve the level of evidence allowing FDG PET/CT to be included in practice guidelines.

\section{References}

1. Brignole M, Auricchio A, Baron-Esquivias G, Bordachar P, Boriani G, Breithardt OA, et al. 2013 ESC guidelines on cardiac pacing and cardiac resynchronization therapy: The Task Force on cardiac pacing and resynchronization therapy of the European Society of Cardiology (ESC). Developed in collaboration with the European Heart Rhythm Association (EHRA). Eur Heart J 2013;34:2281-329.
2. Yancy CW, Jessup M, Bozkurt B, Butler J, Casey DE Jr, Drazner $\mathrm{MH}$, et al. 2013 ACCF/AHA guideline for the management of heart failure: A report of the American College of Cardiology Foundation/American Heart Association Task Force on practice guidelines. Circulation 2013;128:e240-327.

3. Nof E, Epstein LM. Complications of cardiac implants: Handling device infections. Eur Heart J 2013;34:229-36.

4. Bensimhon L, Lavergne T, Hugonnet F, Mainardi JL, Latremouille C, Maunoury C, et al. Whole body [(18) F]fluorodeoxyglucose positron emission tomography imaging for the diagnosis of pacemaker or implantable cardioverter defibrillator infection: A preliminary prospective study. Clin Microbiol Infect 2011;17:836-44.

5. Ploux S, Riviere A, Amraoui S, Whinnett Z, Barandon L, Lafitte S, et al. Positron emission tomography in patients with suspected pacing system infections may play a critical role in difficult cases. Heart Rhythm 2011;8:1478-81.

6. Cautela J, Alessandrini S, Cammilleri S, Giorgi R, Richet H, Casalta JP, et al. Diagnostic yield of FDG positron-emission tomography/computed tomography in patients with CEID infection: A pilot study. Europace 2013;15:252-7.

7. Sarrazin JF, Philippon F, Tessier M, Guimond J, Molin F, Champagne $\mathrm{J}$, et al. Usefulness of fluorine-18 positron emission tomography/computed tomography for identification of cardiovascular implantable electronic device infections. J Am Coll Cardiol 2012;59:1616-25.

8. Leccisotti L, Perna F, Lago M, Leo M, Stefanelli A, Calcagni ML, et al. Cardiovascular implantable electronic device infection: Delayed vs standard FDG PET-CT imaging. J Nucl Cardiol 2014;21:622-32.

9. Tlili G, Amraoui S, Mesguish C, Riviere A, Bordachar P, Hindié E, et al. High performances of $18 \mathrm{~F}$-fluorodeoxyglucose PET-CT in cardiac implantable device infections: A study of 40 patients. J Nucl Cardiol 2015. doi:10.1007/s12350-015-0067-x.

10. Ohira H, Tsujino I, Yoshinaga K. (1)(8)F-Fluoro-2-deoxyglucose positron emission tomography in cardiac sarcoidosis. Eur J Nucl Med Mol Imaging 2011;38:1773-83.

11. Kouijzer IJ, Vos FJ, Janssen MJ, van Dijk AP, Oyen WJ, BleekerRovers CP. The value of 18F-FDG PET/CT in diagnosing infectious endocarditis. Eur J Nucl Med Mol Imaging 2013;40:1102-7.

12. Saby L, Laas O, Habib G, Cammilleri S, Mancini J, Tessonnier L, et al. Positron emission tomography/computed tomography for diagnosis of prosthetic valve endocarditis: Increased valvular 18Ffluorodeoxyglucose uptake as a novel major criterion. J Am Coll Cardiol 2013;61:2374-82.

13. Baddour LM, Epstein AE, Erickson CC, Knight BP, Levison ME, Lockhart PB, et al. Update on cardiovascular implantable electronic device infections and their management: A scientific statement from the American Heart Association. Circulation 2010;121:458-77.

14. Erba PA, Conti U, Lazzeri E, Sollini M, Doria R, De Tommasi $\mathrm{SM}$, et al. Added value of $99 \mathrm{mTc}-\mathrm{HMPAO}-$ labeled leukocyte SPECT/CT in the characterization and management of patients with infectious endocarditis. J Nucl Med 2012;53:1235-43.

15. Hyafil F, Rouzet F, Lepage L, Benali K, Raffoul R, Duval X, et al. Role of radiolabelled leucocyte scintigraphy in patients with a suspicion of prosthetic valve endocarditis and inconclusive echocardiography. Eur Heart J Cardiovasc Imaging 2013;14:58694.

16. Erba PA, Sollini M, Conti U, Bandera F, Tascini C, De Tommasi $\mathrm{SM}$, et al. Radiolabeled WBC scintigraphy in the diagnostic workup of patients with suspected device-related infections. JACC Cardiovasc Imaging 2013;6:1075-86.

17. Rouzet F, Chequer R, Benali K, Lepage L, Ghodbane W, Duval X, et al. Respective performance of 18F-FDG PET and radiolabeled 
leukocyte scintigraphy for the diagnosis of prosthetic valve endocarditis. J Nucl Med 2014;55:1980-5.

18. Vos FJ, Bleeker-Rovers CP, Kullberg BJ, Adang EM, Oyen WJ. Cost-effectiveness of routine (18)F-FDG PET/CT in high-risk patients with gram-positive bacteremia. J Nucl Med 2011;52:1673-8.
19. Iung B, Erba PA, Petrosillo N, Lazzeri E. Common diagnostic flowcharts in infective endocarditis. Q J Nucl Med Mol Imaging 2014;58:55-65. 